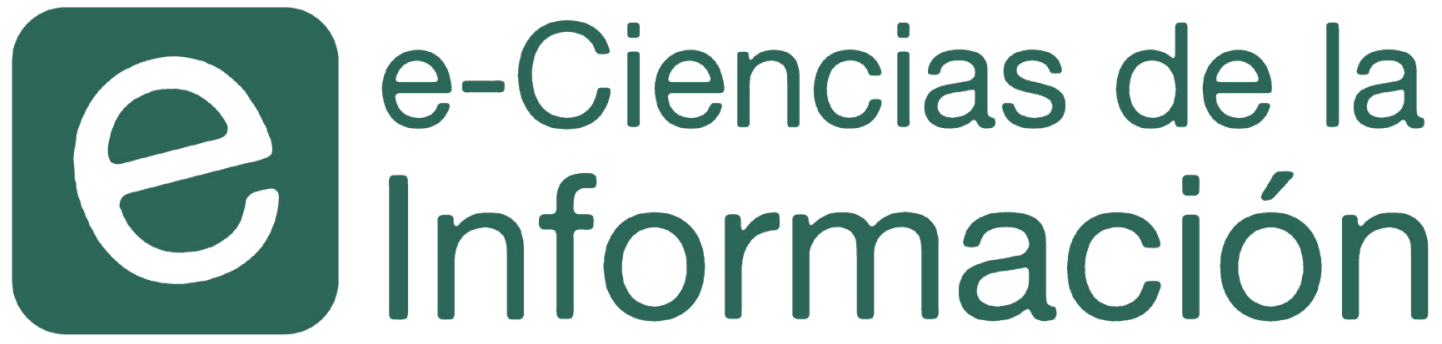

\title{
Análisis bibliométrico de los proyectos de graduación de ingeniería eléctrica de la Universidad de Costa Rica 1999-2018
}

\section{Marvin Coto Jiménez}

Recibido: 5/08/2019 | Corregido: 21/10/2019 | Aceptado: 10/11/2019 DOI:10.15517/eci.v10i1.39883

e-Ciencias de la Información, volumen 10, número 2, Ene- Jun 2020

ISSN: 1649-4142

\section{다(1)(5)(2)}

\section{¿Cómo citar este artículo?}

Coto Jiménez, M. (2020). Análisis bibliométrico de los proyectos de graduación de ingeniería eléctrica de la Universidad de Costa Rica 1999-2018. e-Ciencias de la Información, 10(1). Doi: 10.15517/eci. 


\title{
Análisis bibliométrico de los proyectos de graduación de ingeniería eléctrica de la Universidad de Costa Rica 1999-2018
}

\author{
Bibliometric analysis of the graduation projects in \\ electrical engineering of the University of Costa Rica
}

1999-2018

\author{
Marvin Coto Jiménez (iD ${ }^{1}$
}

\section{RESUMEN}

El objetivo de esta investigación fue realizar un análisis bibliométrico de los proyectos de graduación de pregrado en la Escuela de Ingeniería Eléctrica de la Universidad de Costa Rica, entre los años 1999 y 2018. El interés principal es desarrollar un análisis descriptivo de la distribución temporal y temática de estos documentos, los cuales se realizan de forma individual por los estudiantes de esta carrera para optar por el título de Bachillerato en Ingeniería Eléctrica. En tal sentido, se analizaron las siguientes variables: a) número de proyectos por quinquenio, b) áreas temáticas, c) número de páginas por documento, d) género de los autores. Se obtuvo un total de 938 documentos, utilizando la información contenida en las bases de datos del Sistema de Bibliotecas, Documentación e Información de la Universidad de Costa Rica. Los resultados se discutieron en términos de la evolución de las variables y temáticas a lo largo de los veinte años del período estudiado. Entre los hallazgos se encuentran la mayor participación de las mujeres y la reducción del tamaño de los documentos en años recientes, así como la constante actualización de las temáticas. Estos resultados son un reflejo de los intereses y áreas activas de esta carrera, por lo que los resultados pueden constituir un indicador de la evolución de la disciplina de Ingeniería Eléctrica en la institución.

Palabras Clave: Bibliometría, Ingeniería Eléctrica, proyecto del alumno, proyectos de investigación.

\section{Abstract}

The purpose of this research was to perform a bibliometric analysis of undergraduate graduation projects at the School of Electrical Engineering of the University of Costa Rica, between the years 1999 and 2018. The main interest is to perform a descriptive analysis of the temporal distribution and thematic areas of these documents, which are carried out individually by the students of this career to opt for the Bachelor's Degree in Electrical Engineering. To perform 
the analysis, the following variables were obtained: a) number of projects per quinquennium, b) thematic areas, c) number of pages per document, d) gender of the authors. In total, 938 documents were analyzed, using the information contained in the databases of the SIBDI system of the University of Costa Rica. The results were discussed in terms of the evolution of the variables and themes throughout the twenty years of the period studied. Among the findings are the greater participation of women in recent years, the reduction of the size of the documents, and the constant updating of the themes. These results reflect the interests and active areas of this career, so the results can be an indicator of the evolution of the Electrical Engineering discipline in the institution.

Key Words: Bibliometry, Electrical Engineering, student projects, research projects.

\section{Introducción}

El conocimiento generado por las diversas áreas del quehacer científico es usualmente difundido a través de artículos, libros, tesis, actas de congresos, entre otros, en los cuales se marca la productividad y características de los grupos específicos que los generan. Para aprovechar esta información, han surgido los estudios bibliométricos como instrumentos que aportan datos importantes para valorar la situación de áreas de conocimiento específicas, y contar así con análisis cualitativos y cuantitativos que permitan verificar su estado y la productividad de quienes los desarrollan.

Conceptualmente, la bibliometría ha sido definida como "la aplicación de métodos matemáticos a la literatura científica, fundamentados en la estadística y los índices bibliométricos." (Corrales-Reyes, Fornaris-Cedeno, y Reyes-Pérez, 2018, p. 19). En cuanto a su utilidad, como indica Nicaragua (2013, p.2), citando a Borbons y Zulueta (1999, p.790)

Losestudiosbibliométricosseencargandeanalizar,cuantitativamente, la producción científica y, además, complementan de manera eficaz las opiniones y los juicios emitidos por los expertos de cada área proporcionando herramientas útiles y objetivas en los procesos de evaluación de los resultados de la actividad científica.

Desde las ciencias de la información, se ha reconocido que "las publicaciones científicas reflejan la investigación realizada en todos los niveles del conocimiento por grupos o centros de investigación" (Maz-Machado, Jiménez-Fanjul y Villagra-Rico, 2016, p.112). Su utilidad se ve reflejada en análisis de la evolución en el tiempo, productividad, colaboración, impacto y visibilidad, entre otros (Ferrer-Villalobos, Freire-Constante, SuasnavasBermúdez, Merino-Salazar y Gómez-García, 2017).

Entre la producción científica característica de la Escuela de Ingeniería Eléctrica de la Universidad de Costa Rica están los proyectos eléctricos, los cuales tienen como finalidad la integración de estrategias de diseño e investigación dentro de una temática abierta, usualmente propuesta por los propios profesores. Estos proyectos culminan con un trabajo escrito que cuenta con un formato preestablecido, el cual incluye un compendio de la teoría, el diseño o análisis particular del problema propuesto, y los resultados del proyecto. El proyecto es integrador, abierto en su temática y de aplicación de estrategias de investigación (Romero Chacón 2018). 
Las características de los proyectos eléctricos, requisito para obtener el grado de Bachillerato en Ingeniería Eléctrica, se asemejan a una tesis, aunque las normas y el rigor exigido es menor al establecido en trabajos finales de graduación de licenciatura o de posgrado en el área. Desde el punto de vista del currículo se matricula como una materia, por lo que debe desarrollarse durante las 16 semanas de un ciclo normal, con opción a solicitar una prórroga en caso de solicitarlo el estudiante.

Dado que se realizan de forma individual, y por su carácter obligatorio para obtener el grado de bachillerato (con una malla curricular de 8 semestres), como objeto de análisis es provechoso, pues constituye un reflejo de las líneas, tendencias y potencialidades de la investigación e intereses del área, como otros documentos propios del final de un proceso de estudios.

En el presente trabajo se realiza un estudio bibliométrico de los proyectos eléctricos realizados en la Escuela de Ingeniería Eléctrica de la Universidad de Costa Rica entre los años 1999 a 2018, con el objetivo de cuantificar y trazar cronológicamente la producción de estos trabajos, y de esta manera ofrecer un panorama de los cambios en las temáticas y características que se han producido en la evolución de esta área del conocimiento reflejado.

Para esto, específicamente se ha partido de una base de datos de acceso público donde se puede acceder a la totalidad de registros de información de estos documentos, y se implementaron varias etapas de procesamiento para depurar y presentar los resultados. Este procedimiento y presentación de resultados ha sido acorde con referencias recientes de estudios bibliométricos en distintas áreas del conocimiento.

Se considera de interés los resultados de este trabajo por el aporte que puede ofrecer para docentes, profesionales en el área, académicos e investigadores, ya que la información analizada abarca un período de veinte años, en el cual pocos estudios han caracterizado la especificidad de la Ingeniería Eléctrica en Costa Rica.

\section{Referente teórico}

De acuerdo con Rueda-Clausen, Villa-Roel y Rueda-Clausen (2005, p. 29), la bibliometría "nace a partir de la utilización de métodos matemáticos y estadísticos para el análisis de la producción científica y de su calidad". El autor Eugene Garfield la definió inicialmente como "la cuantificación de la información bibliográfica susceptible de ser analizada" (Rueda-Clausen et al., 2005, p. 29).

La cantidad de estudios bibliométricos realizados en distintas áreas ha ido en aumento desde su establecimiento (Ferrada, Díaz-Levicoy, Salgado Orellana y Puraivan, 2019), por la necesidad de analizar el desarrollo de la producción científica, y la caracterización de los campos donde se desarrolla investigación.

Dada la amplitud y la diferenciación que puede lograrse en estos análisis de la producción científica, se han propuesto una diversidad de indicadores para lograr una valoración adecuada de acuerdo con el tipo de publicación. Por ejemplo, se cuenta con indicadores de contemporaneidad, productividad y factor de impacto cuando es posible medir aspectos como la cantidad de 
citas generadas a partir de una publicación. El análisis de citas para evaluar publicaciones ha sido discutido previamente por Garfield y Merton (1979), y se ha consolidado como una métrica útil y objetiva.

Otras métricas han sido utilizadas con fines descriptivos. Por ejemplo, cuando un mismo autor publica varios documentos en un período de tiempo determinado, como el caso de artículos en revistas académicas. Entre ellas el índice de Price (Price, 1965), en relación con la proporción de citas que corresponden a los últimos cinco años de la literatura. O bien, como indica Ardanuy (2012, p.23), existen indicadores de forma y contenido, los cuales toman en cuenta la distribución porcentual de temas, las lenguas utilizadas, el soporte y el porcentaje de documentos de cada tipología documental.

Entre la amplia gama de posibilidades que los estudios de este tipo pueden ofrecer para analizar la producción académica, se encuentran estudios como el de De León-Camero, Solórzano-Muñoz y Valencia (2018), el cual abarca un amplio tema (educación en ingeniería), y publicaciones en un contexto internacional. En otros estudios se delimita el campo de análisis a una sola revista académica o a tesis dentro de un rango específico de años.

En el caso de tesis o proyectos de graduación para obtener grados dentro de instituciones de educación superior, la naturaleza de estas publicaciones hace que usualmente están destinadas a la consulta dentro de los sistemas de documentación de las instituciones que los generan. Por esta razón no se indexan ni difunden dentro de los sistemas tradicionales de publicación, por lo cual no se analizan con este tipo de indicadores. Autores como Mathews (2004) han catalogado este tipo de documentos como "literatura gris", categoría en la que también se incluyen reportes técnicos y documentos de negocios.

En lugar de indicadores relacionados con citas, tal como se muestra en diversas experiencias relacionadas con estudios bibliométricos en tesis y proyectos de graduación como Moreno-Fernández y Moreno-Crespo (2016), Rey y Acevedo (2015), Delgado, Torres-Salinas, Jiménez-Contreras, y RuizPérez (2006), Valle y Salvador (2009), y Castillo y Xifra (2006), las variables que usualmente se analizan son cualitativas, con escala de medición nominal, tales como: año de registro, área de investigación, especialidad, evolución del número de tesis y temática. También se incluye análisis por quinquenios, como Repiso, Torres y Delgado (2011, p.154), quienes también destacan:

El estudio de las tesis doctorales como objeto de análisis es enormemente provechosoya queéstas constituyen uno delosmejores espejos donde se reflejan las líneas, tendencias y potencialidades de la investigación de las universidades. Asimismo, es un medio idóneo para conocer la estructura social de la investigación permitiendo analizar no solo la evolución científica de un área concreta de estudio sino también identificar cuáles son sus principales protagonistas y cómo se relacionan entre sí.

En el caso de tesis de pregrado, Lagos y Pérez-Gutiérrez (2016, p. 183), indican:

En el ámbito de las tesis de pregrado existen varios trabajos que han pretendido establecer mediciones de los procesos al interior de las escuelas de formación universitaria, centrando su interés sobre los trabajos de los estudiantes y la producción científica en distintos ámbitos del conocimiento como la medicina. 
De manera que, tanto para el caso de estudios de posgrado como de pregrado, las tesis y documentos equivalentes como requisito de obtención de grado han sido utilizados como una forma de caracterizar diversos aspectos de las áreas del saber y su evolución. Como indica Aguilar et al. (2007, p.319), "en general son útiles para el conocimiento de los procesos en los centros de enseñanza y la naturaleza de las áreas temáticas".

En algunos casos, se han realizado análisis de toda la producción de tesis en determinada carrera, como Aguilar et al. (2007), Chamba-Herrera, L. (2018), Lagos y Pérez-Gutiérrez, (2016), y en otros casos sobre tesis realizadas sobre temas más específicos Rey y Acevedo (2015), Pineda, Valencia y Roldán, J. (2018). Coincidente con la primera categoría, en el presente trabajo se aborda un análisis bibliométrico de toda la producción de proyectos de graduación del Bachillerato en Ingeniería Eléctrica de la Universidad de Costa Rica.

\section{Método}

\subsection{Tipo de investigación}

Este estudio tuvo un carácter descriptivo bibliométrico cuantitativo. Para su realización se definió una serie de variables por medio de las cuales se analizó el material documental en las bases de datos del Sistema de Bibliotecas, Documentación en Información (SIBDI) de la Universidad de Costa Rica. El SIBDI ofrece un servicio de libre acceso a todos los materiales referenciados en las bibliotecas de esta universidad. La búsqueda permite el uso de palabras clave, autor, y filtros tales como el año, la biblioteca en específico donde se desea hacer la búsqueda, el idioma, entre otros.

\subsection{Universo de Estudio}

El universo de estudio estuvo conformado por todos los documentos registrados como "Proyecto Eléctrico" en las bases de dato mencionados, durante el período 1999-2018. Para la recolección de este material documental se revisó la base de datos del catálogo OPAC del SIBDI entre los años definidos, utilizando las herramientas del sitio web del SIBDI. En este catálogo se encuentran todos los documentos disponibles en las distintas bibliotecas que conforman el SIBDI, y es de acceso abierto.

\subsection{Procedimiento}

El procedimiento general de realización de un estudio bibliométrico ha sido manifiesto en otros estudios, como Moreno-Fernández y Moreno-Crespo (2016), Salas et al. (2017), y De León-Camero et al, (2018). En éstos, se parte de una base de datos que contiene los registros de los documentos de interés para el estudio (ya sea artículos de revista o trabajos de graduación en determinada institución), se establecen los métodos de búsqueda y se analizan los resultados. En ocasiones, como en el mencionado Salas et al. (2017), se trata de bases de datos de libre acceso en Internet. 
El análisis es usualmente un procedimiento sistemático usando un conjunto de indicadores generales (cantidad de documentos, número de páginas, género del autor, entre otros), así como otros aspectos que se puedan determinar o inferir a partir de la información accesible a través de las bases de datos correspondientes.

El procedimiento seguido se esquematiza en la Figura 1. Como primer paso, una vez que en el sistema OPAC se tiene el resultado de la búsqueda, ésta se descarga en formato HTML. Los campos de información a los que se tiene acceso en cada documento, y que son de relevante para el presente estudio son:

1. Signatura: para la localización del documento dentro del Sistema de Bibliotecas.

2. Autor personal: nombre del autor (estudiante de Ingeniería Eléctrica).

3. Título.

4. Pie de imprenta: donde se consiga ciudad, país y año de publicación.

5. Página y otros: contiene la cantidad de páginas del documento.

6. Tesis: donde se indica que el registro corresponde a un proyecto de graduación del bachillerato en Ingeniería Eléctrica.

7. Materia: lista de materias con las cuales el documento está catalogado.

FIGURA 1

Esquema del procedimiento de obtención y depuración de información.

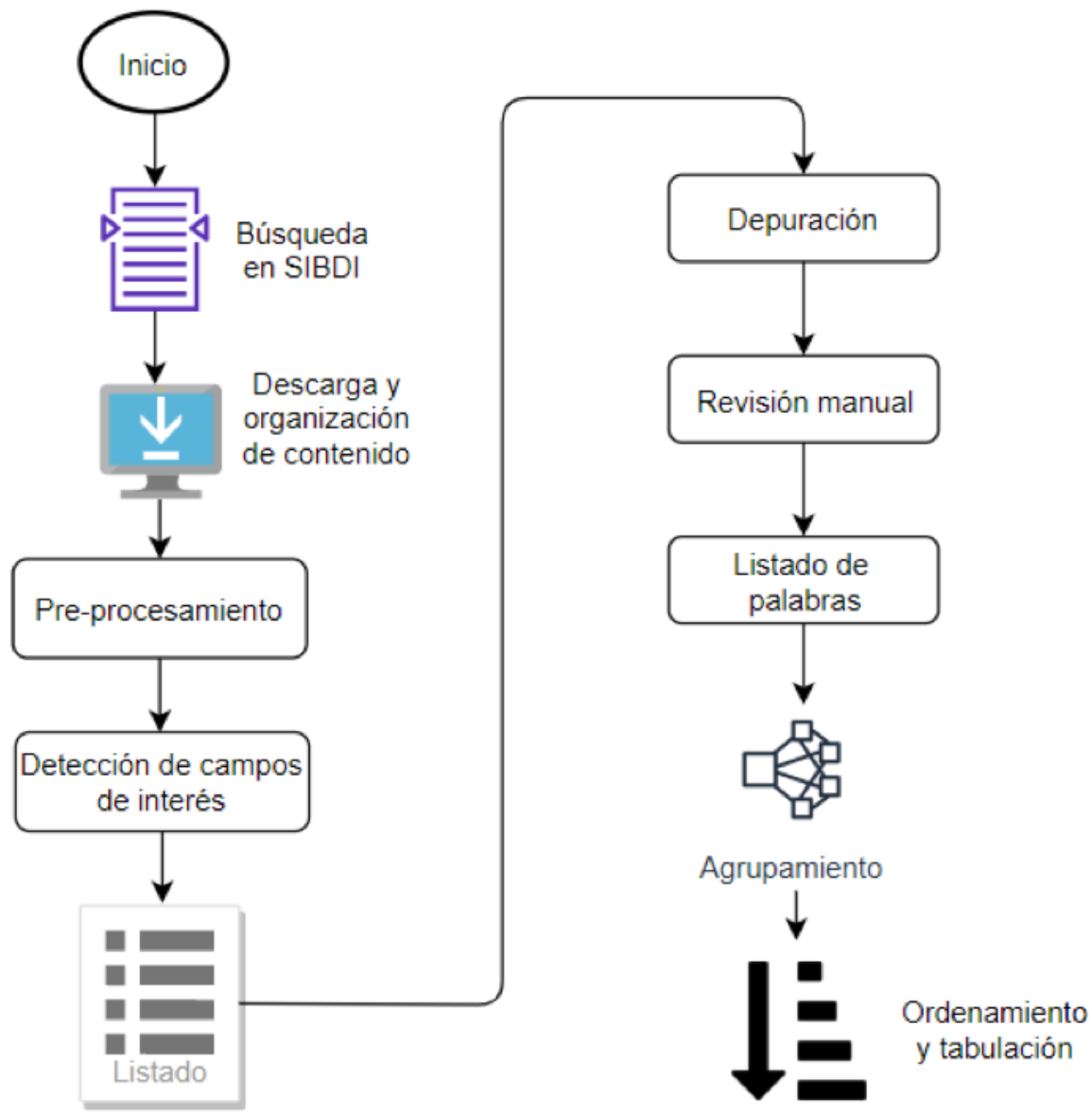

Fuente: Elaboración propia, 2019 
Con los resultados de las búsquedas, se descargaron los resultados correspondientes a cada uno de los quinquenios definidos en el estudio: 1999-2003, 2004-2008, 2009-2013, 2014-2018. Dado que estos resultados están en formato HTML, contienen gran cantidad de código para el correcto despliegue de la información en la página de Internet. Por esta razón, la extracción automatizada de información requirió el desarrollo de algunos programas que aprovecharan herramientas informáticas de procesamiento de archivos de texto.

Si bien existen herramientas ya desarrolladas para este fin (como el sistema Stopwords), para el presente trabajo se optó por la generación de software específico para los archivos obtenidos y la información que se deseaba extraer.En particular, en el sistema operativo Linux se cuenta con sistemas como $a w k^{2}$, sed ${ }^{3}$ y sort ${ }^{4}$, con los cuales se pueden buscar líneas específicas dentro de documentos HTML, eliminar información no útil, y localizar aquella que lo sea para los fines de la investigación. Se desarrollaron algunas rutinas de programación dentro del lenguaje bash del sistema operativo para localizar, extraer y listar los campos de interés.

Por ejemplo, la información es usualmente contenida entre segmentos específicos del código HTML, entre indicadores como <td class=td1> Materia \&nbsp; $</$ td $>$ y $</$ span $>$.

Los programas desarrollados tienen como salida archivos con listas de los campos de interés, por ejemplo, la lista de títulos o de autores. Dado que estas listas aún contienen fragmentos de código y símbolos que no son útiles para el procesamiento automático, se utilizaron otras herramientas (como egrep y sort), para depurar los símbolos y homogenizar las palabras en cuanto a minúsculas, para evitar duplicidades en el procesamiento que distinguieran palabras como "sistema" $y$ "Sistema".

Luego de la utilización de estas herramientas informáticas, el siguiente paso requirió la inspección manual de los resultados, para eliminar aquellos símbolos que hubieran quedado de los procesos anteriores, puntuación y otros, a la vez que se revisó que no se hubieran eliminado símbolos que sí son de importancia para el área de estudio, como los símbolos de suma en " $\mathrm{C}++$ " (lenguaje informático), que lo diferencian del lenguaje llamado " $C$ ".

Para el caso del análisis de palabras en los títulos, nuevos procedimientos fueron necesarios para hacer listado de palabras en las listas generadas, y la contabilización automática de la cantidad de veces que aparecen las palabras. En los listados, fue importante el agrupamiento de algunos términos semejantes (como sistema/sistemas; o microcontrolador/ microcontroladores), los cuales representan el mismo concepto.

\footnotetext{
ªwk es el nombre del programa y lenguaje de programación diseñado para manipular y seleccionar secciones particulares de archivos de texto. Es parte usual de las distribuciones del sistema operativo Linux. Disponible en: https://www.gnu.org/software/gawk/gawk.html

${ }^{3}$ sed es una herramienta de software implementada en el sistema operativo Linux para modificar líneas o campos específicos de archivos de texto, de acuerdo con una rutina escrita por el usuario, establecida en un lenguaje de programación propio. Disponible en: http://sed.sourceforge.net/sedfaq2.html\#s2.1

${ }^{4}$ sort es el nombre de un programa implementado en el sistema operativo Linux, el cual permite ordenar archivos de texto de acuerdo con los criterios establecidos por el usuario en determinado lenguaje. Disponible en: $\underline{\text { http://man7.org/linux/man-pages/man1/sort.1.html }}$
} 
Finalmente, la información procesada de esta manera fue listada y tabulada de forma manual, lo que permitió eliminar o corregir, según correspondiera, artículos, preposiciones y conjunciones que no aportaran al análisis requerido.

\section{Resultados y discusión}

\subsection{Cantidad de documentos}

En la Tabla 1 se muestra la cantidad de Proyectos Eléctricos registrados entre los años 1999 y 2018, agrupados por quinquenio. El total de documentos analizados es de 938.

TABLA 1

Número de documentos presentados como proyecto eléctrico por quinquenios, extraídos de la base de datos del SIBDI, Escuela de Ingeniería Eléctrica 1999-2018.

\begin{tabular}{lcc}
\hline \multicolumn{1}{c}{ QUINQUENIO } & $\begin{array}{c}\text { FRECUENCIA } \\
\text { ABSOLUTA }\end{array}$ & FRECUENCIA RELATIVA \\
\hline $1999-2003$ & 181 & $19.3 \%$ \\
$2004-2008$ & 226 & $24.1 \%$ \\
$2009-2013$ & 308 & $32.8 \%$ \\
$2014-2018$ & 223 & $23.8 \%$ \\
Total: & 938 & $100 \%$ \\
\hline
\end{tabular}

Fuente: Elaboración propia, 2019.

Se puede observar, acorde con el crecimiento de la cantidad de estudiantes en la carrera durante el período de estudio, un incremento en la cantidad de proyectos presentados entre los años 1999 y 2003, con una disminución para el período 2014-2018, que la asemeja al quinquenio 2004-2008. El quinquenio 2009-2013 es el que presenta mayor cantidad de proyectos de graduación en el período estudiado, con un 32.8\% del total.

\subsection{Longitud promedio de los documentos}

La estructuración de los documentos de Proyecto Eléctrico ha pasado por distintos períodos, en los cuales se refleja la evolución de la tecnología de procesamiento de texto. De esta manera, se tienen, como otros documentos de proyectos de graduación en la Universidad de Costa Rica, documentos en formato digital e impresos con los primeros procesadores ampliamente difundidos, hasta los actuales que se basan en el procesador LaTeX con una plantilla definida por la Escuela.

Esta nueva plantilla puede ser la explicación por la cual los documentos de proyectos eléctricos han tendido a disminuir la cantidad de páginas 
promedio, lo cual ha sido reportado en estudios semejantes como indicador de la extensión y profundidad del requerimiento característica de las tesis. Por ejemplo, Chamba-Herrera (2018, p. 112) indica que, para el caso de Ingeniería Forestal en la Universidad de Loja, Ecuador, entre los años 2013-2017, el 83\% de las tesis contiene entre 61 y 120 páginas.

Para el caso de Ingeniería Eléctrica en la Universidad de Costa Rica, se tiene el máximo de 98 páginas promedio (desviación estándar 46) en el quinquenio 2004-2008, mientras que en el quinquenio 2014-2018 han disminuido a 65 (desviación estándar 31), como se aprecia en la Tabla 2. La reducción del último quinquenio es significativa con respecto a los anteriores, así como la desviación estándar. En comparación con el caso de Ingeniería Forestal mencionado anteriormente, los documentos en la Universidad de Costa Rica tienden a ser de longitud comparable, aunque no se cuenta con datos precisos para hacer una comparación directa.

\section{TABLA 2}

Longitud promedio de documentos presentados como proyecto eléctrico por quinquenios, extraídos de la base de datos del SIBDI, Escuela de Ingeniería

\section{Eléctrica 1999-2018}

\begin{tabular}{|c|c|c|}
\hline QUINQUENIO & $\begin{array}{l}\text { PROMEDIO DE } \\
\text { NÚMERO DE PÁGINAS }\end{array}$ & DESVIACIÓN ESTÁNDAR \\
\hline $1999-2003$ & 93 & 53 \\
\hline 2004-2008 & 98 & 46 \\
\hline 2009-2013 & 94 & 54 \\
\hline 2014-2018 & 65 & 31 \\
\hline
\end{tabular}

Fuente: Elaboración propia, 2019.

Dado que en 1999 aún no se contaba con sistemas informáticos de amplia difusión, la uniformidad de un formato que contemple tipos y tamaños de letra, y secciones pre-definidas no fue posible sino hasta años recientes. De contarse con un indicador como número de palabras en el documento, se tendría un indicador para establecer una mayor comparación. Sin embargo, este descriptor no se encuentra disponible en las bases de datos consultadas.

\subsection{Género de los autores}

Es sabido que la carrera de Ingeniería Eléctrica ha tenido históricamente una predominancia de varones, con un repunto de participación femenina en los últimos años. De hecho, como se observa en la Tabla 3, el mayor porcentaje de mujeres que presentaron su proyecto de graduación en los últimos veinte años lo hicieron en el quinquenio 2014-2018. En éste, se tuvo un $15.7 \%$ de mujeres, en comparación con un $11.6 \%$ (participación mínima del período en estudio) en el quinquenio 1999-2003. 
TABLA 3

Género del autor principal de documentos presentados como proyecto eléctrico por quinquenios, extraídos de la base de datos del SIBDI, Escuela de Ingeniería Eléctrica 1999-2018

\begin{tabular}{lcccc}
\hline \multicolumn{1}{c}{ QUINQUENIO } & MUJERES & \% MUJERES & HOMBRES & \% HOMBRES \\
\hline $1999-2003$ & 21 & $11.6 \%$ & 160 & $88.4 \%$ \\
$2004-2008$ & 33 & $14.6 \%$ & 193 & $85.4 \%$ \\
$2009-2013$ & 43 & $14.0 \%$ & 265 & $86.0 \%$ \\
$2014-2018$ & 35 & $15.7 \%$ & 188 & $84.3 \%$ \\
Total: & 132 & $14.1 \%$ & 806 & $85.9 \%$ \\
\hline
\end{tabular}

Fuente: Elaboración propia, 2019.

En los veinte años que abarca el período de estudio, la participación de las mujeres en la generación de proyectos eléctricos es de un $14.1 \%$. Este porcentaje de participación de mujeres es menor al presentado en otras áreas de la ingeniería analizados en América Latina recientemente, como el caso de ingeniería forestal (Chamba-Herrera, 2018, p.111).

En la Figura 2 se observa la tendencia del número total de proyectos eléctricos y del género de sus autores.

FIGURA 2

Tendencia de cantidad de proyectos totales por quinquenio y por género del autor

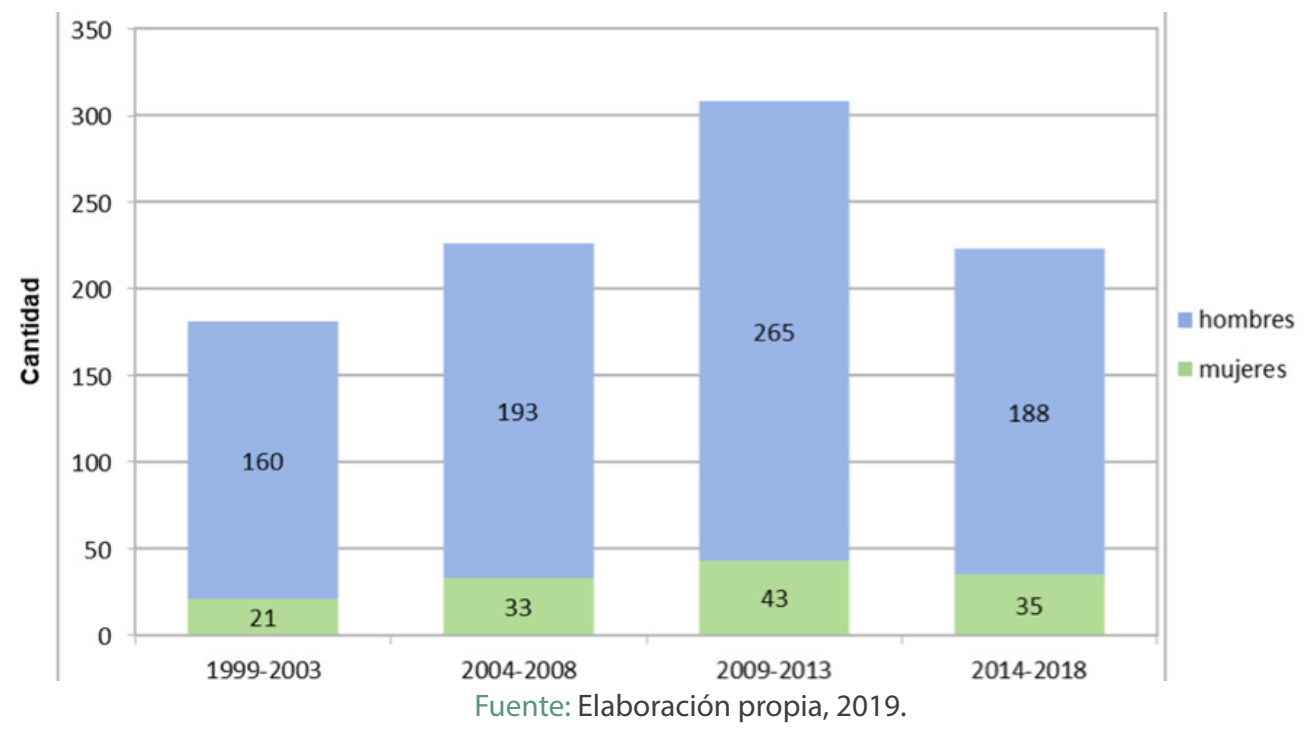

\subsection{Principales áreas temáticas}

En la Tabla 4 se muestra un ordenamiento de las principales áreas temáticas presentes en los proyectos de graduación del bachillerato en Ingeniería Eléctrica. Éstos fueron obtenidos del campo "materia" en la clasificación del SIBDI. Las tres principales áreas temáticas son: Distribución de energía eléctrica, Sistemas de energía eléctrica y Control automático. 
TABLA 4

Áreas temáticas de los documentos presentados como proyecto eléctrico por quinquenios, extraídos del campo "materia" en la base de datos del SIBDI, Escuela de Ingeniería Eléctrica 1999-2018

\begin{tabular}{|c|c|c|c|c|c|c|}
\hline MATERIA & 1999-2003 & 2004-2008 & 2009-2013 & 2014-2018 & TOTAL & $\begin{array}{c}\text { FREC. } \\
\text { RELATIVA }\end{array}$ \\
\hline $\begin{array}{l}\text { Distribución de } \\
\text { energía eléctrica }\end{array}$ & 15 & 23 & 86 & 40 & 164 & $11.25 \%$ \\
\hline $\begin{array}{c}\text { Control } \\
\text { automático }\end{array}$ & 31 & 26 & 65 & 19 & 141 & $6.67 \%$ \\
\hline $\begin{array}{c}\text { Sistemas de } \\
\text { energía eléctrica }\end{array}$ & 4 & 18 & 44 & 23 & 89 & $6.10 \%$ \\
\hline $\begin{array}{c}\text { Sistemas de } \\
\text { transmisión de } \\
\text { datos }\end{array}$ & 19 & 25 & 21 & 13 & 78 & $5.35 \%$ \\
\hline $\begin{array}{c}\text { Simulación por } \\
\text { computadora }\end{array}$ & 12 & 6 & 18 & 29 & 65 & $4.46 \%$ \\
\hline $\begin{array}{c}\text { Programas para } \\
\text { computadora }\end{array}$ & 26 & 9 & 19 & 2 & 56 & $3.84 \%$ \\
\hline $\begin{array}{l}\text { Controladores } \\
\text { lógicos } \\
\text { programables }\end{array}$ & 11 & 4 & 23 & 11 & 49 & $3.36 \%$ \\
\hline Controladores PID & 10 & 11 & 16 & 7 & 44 & $3.02 \%$ \\
\hline Energía eléctrica & 4 & 4 & 25 & 9 & 42 & $2.88 \%$ \\
\hline Diseño & 27 & 13 & 0 & 0 & 40 & $2.74 \%$ \\
\hline $\begin{array}{c}\text { Interfases } \\
\text { con el usuario } \\
\text { (sistema para } \\
\text { computadora) }\end{array}$ & 11 & 10 & 9 & 10 & 40 & $2.74 \%$ \\
\hline $\begin{array}{l}\text { Sistemas de } \\
\text { telecomunicación }\end{array}$ & 3 & 14 & 18 & 5 & 40 & $2.74 \%$ \\
\hline $\begin{array}{l}\text { Instalaciones } \\
\text { eléctricas }\end{array}$ & 5 & 6 & 13 & 16 & 40 & $2.74 \%$ \\
\hline $\begin{array}{l}\text { Transformadores } \\
\text { eléctricos }\end{array}$ & 4 & 6 & 20 & 6 & 36 & $2.47 \%$ \\
\hline $\begin{array}{l}\text { Procesamiento de } \\
\text { señales }\end{array}$ & 13 & 6 & 10 & 6 & 35 & $2.40 \%$ \\
\hline $\begin{array}{l}\text { Sistemas de } \\
\text { control }\end{array}$ & 2 & 6 & 10 & 16 & 34 & $2.33 \%$ \\
\hline $\begin{array}{l}\text { Sistemas móviles } \\
\text { de comunicación }\end{array}$ & 1 & 15 & 12 & 5 & 33 & $2.26 \%$ \\
\hline
\end{tabular}




\begin{tabular}{|c|c|c|c|c|c|c|}
\hline MATERIA & 1999-2003 & $2004-2008$ & $2009-2013$ & 2014-2018 & TOTAL & $\begin{array}{c}\text { FREC. } \\
\text { RELATIVA }\end{array}$ \\
\hline $\begin{array}{l}\text { Reguladores } \\
\text { eléctricos }\end{array}$ & 9 & 11 & 10 & 2 & 32 & $2.19 \%$ \\
\hline $\begin{array}{l}\text { Transmisión de } \\
\text { potencia }\end{array}$ & 2 & 9 & 13 & 6 & 30 & $2.06 \%$ \\
\hline Automatización & 11 & 6 & 10 & 2 & 29 & $1.99 \%$ \\
\hline $\begin{array}{l}\text { Sistemas de } \\
\text { control digital }\end{array}$ & 4 & 11 & 11 & 3 & 29 & $1.99 \%$ \\
\hline $\begin{array}{l}\text { Circuitos de } \\
\text { interfaz }\end{array}$ & 9 & 2 & 11 & 5 & 27 & $1.85 \%$ \\
\hline $\begin{array}{l}\text { Redes eléctri- } \\
\text { cas }\end{array}$ & 3 & 3 & 10 & 9 & 25 & $1.71 \%$ \\
\hline $\begin{array}{c}\text { Circuitos eléc- } \\
\text { tricos }\end{array}$ & 3 & 3 & 10 & 8 & 24 & $1.65 \%$ \\
\hline $\begin{array}{l}\text { Telecomunica- } \\
\text { ciones }\end{array}$ & 3 & 10 & 6 & 4 & 23 & $1.58 \%$ \\
\hline $\begin{array}{c}\text { Dispositivos } \\
\text { de distribu- } \\
\text { ción }\end{array}$ & 2 & 1 & 18 & 1 & 22 & $1.51 \%$ \\
\hline Dinamos & 4 & 10 & 7 & 0 & 21 & $1.44 \%$ \\
\hline $\begin{array}{c}\text { Generación de } \\
\text { energía }\end{array}$ & 0 & 0 & 12 & 9 & 21 & $1.44 \%$ \\
\hline $\begin{array}{c}\text { Redes de } \\
\text { telecomunica- } \\
\text { ciones }\end{array}$ & 2 & 6 & 10 & 3 & 21 & $1.44 \%$ \\
\hline $\begin{array}{l}\text { Microcontro- } \\
\text { ladores }\end{array}$ & 2 & 3 & 5 & 11 & 21 & $1.44 \%$ \\
\hline $\begin{array}{c}\text { CPUCR } \\
\text { (Microproce- } \\
\text { sador) }\end{array}$ & 12 & 4 & 2 & 2 & 20 & $1.37 \%$ \\
\hline $\begin{array}{c}\text { Electrónica } \\
\text { digital }\end{array}$ & 2 & 0 & 12 & 6 & 20 & $1.37 \%$ \\
\hline $\begin{array}{l}\text { Líneas eléctri- } \\
\text { cas }\end{array}$ & 3 & 4 & 11 & 2 & 20 & $1.37 \%$ \\
\hline $\begin{array}{c}\text { MATLAB } \\
\text { (Programa } \\
\text { para computa- } \\
\text { dora) }\end{array}$ & 0 & 0 & 9 & 10 & 19 & $1.30 \%$ \\
\hline Arduino & 0 & 0 & 1 & 15 & 16 & $1.10 \%$ \\
\hline Internet & 0 & 10 & 2 & 0 & 12 & $0.82 \%$ \\
\hline
\end{tabular}


De la misma Tabla 4 se pueden extraer algunas comparaciones importantes en cuanto a los temas. Por ejemplo, en el quinquenio 1999-2003 se tenía un auge de proyectos basados en el procesador CPUCR, creado en la Escuela de Ingeniería Eléctrica para fines educativos. Después del año 2003, se vio una disminución sustantiva en esta temática, a favor de otras tecnologías de placas de desarrollo de software como Arduino, creado en el año 2005 y que tiene un auge en los proyectos eléctricos desde el quinquenio 2009-2013.

Algunos temas como las interfaces de usuario y los controladores PID han mantenido su presencia de forma constante durante los últimos veinte años. Es importante indicar que en la Tabla 4 se reportan solamente las áreas temáticas que tienen un número de documentos mayor a 12 en el período de estudio, dada la gran longitud que representaría la lista completa.

En la Tabla 5 se muestra un ordenamiento de las materias más frecuentemente utilizadas en cada quinquenio. El control automático fue el más abordado en los quinquenios 1999-2003 y 2004-2008. Posteriormente fue reemplazado como el tema más frecuente por Distribución de energía eléctrica durante los siguientes diez años. Se puede observar cómo el CPUCR se encuentra entre los principales en el período 1999-2003, pero no aparece en la lista de los siguientes quinquenios. Por su parte, la placa de desarrollo Arduino tiene predominancia en el último quinquenio. Los controladores lógicos programables (o PLC, por sus siglas en inglés) han estado presentes casi invariantemente en los temas durante los veinte años del período de estudio.

TABLA 5

Áreas temáticas ordenadas por importancia en de los documentos presentados como proyecto eléctrico por quinquenios, extraídos del campo "materia" en la base de datos del SIBDI, Escuela de Ingeniería Eléctrica 1999-2018

\begin{tabular}{|c|c|c|c|}
\hline 1999-2003 & 2004-2008 & $2009-2013$ & $2014-2018$ \\
\hline Control automático & Control automático & $\begin{array}{c}\text { Distribución de energía } \\
\text { eléctrica }\end{array}$ & $\begin{array}{c}\text { Distribución de energía } \\
\text { eléctrica }\end{array}$ \\
\hline Diseño & $\begin{array}{l}\text { Sistemas de } \\
\text { transmisión de datos }\end{array}$ & Control automático & $\begin{array}{l}\text { Simulación por } \\
\text { computadora }\end{array}$ \\
\hline $\begin{array}{l}\text { Programas para } \\
\text { computadora }\end{array}$ & $\begin{array}{l}\text { Distribución de } \\
\text { energía eléctrica }\end{array}$ & $\begin{array}{c}\text { Sistemas de energía } \\
\text { eléctrica }\end{array}$ & $\begin{array}{c}\text { Sistemas de energía } \\
\text { eléctrica }\end{array}$ \\
\hline $\begin{array}{l}\text { Sistemas de } \\
\text { transmisión de } \\
\text { datos }\end{array}$ & $\begin{array}{c}\text { Sistemas de energía } \\
\text { eléctrica }\end{array}$ & Energía eléctrica & Control automático \\
\hline $\begin{array}{l}\text { Distribución de } \\
\text { energía eléctrica }\end{array}$ & $\begin{array}{l}\text { Sistemas móviles de } \\
\text { comunicación }\end{array}$ & $\begin{array}{c}\text { Controladores lógicos } \\
\text { programables }\end{array}$ & Instalaciones eléctricas \\
\hline $\begin{array}{l}\text { Procesamiento de } \\
\text { señales }\end{array}$ & $\begin{array}{l}\text { Sistemas de } \\
\text { telecomunicación }\end{array}$ & $\begin{array}{c}\text { Sistemas de transmisión } \\
\text { de datos }\end{array}$ & Sistemas de control \\
\hline $\begin{array}{l}\text { Simulación por } \\
\text { computadora }\end{array}$ & Diseño & $\begin{array}{l}\text { Transformadores } \\
\text { eléctricos }\end{array}$ & Arduino \\
\hline $\begin{array}{c}\text { CPUCR } \\
\text { (Microprocesador) }\end{array}$ & Controladores PID & $\begin{array}{l}\text { Programas para } \\
\text { computadora }\end{array}$ & $\begin{array}{c}\text { Sistemas de transmisión de } \\
\text { datos }\end{array}$ \\
\hline $\begin{array}{l}\text { Controladores } \\
\text { lógicos } \\
\text { programables }\end{array}$ & Reguladores eléctricos & $\begin{array}{l}\text { Sistemas de } \\
\text { telecomunicación }\end{array}$ & $\begin{array}{c}\text { Controladores lógicos } \\
\text { programables }\end{array}$ \\
\hline $\begin{array}{l}\text { Interfases con el } \\
\text { usuario (sistema } \\
\text { para computadora) }\end{array}$ & $\begin{array}{c}\text { Sistemas de control } \\
\text { digital }\end{array}$ & $\begin{array}{l}\text { Simulación por } \\
\text { computadora }\end{array}$ & Microcontroladores \\
\hline
\end{tabular}


Los títulos de estos documentos también pueden utilizarse para aquellos conceptos que caracterizan la actividad docente, de investigación e interés general en la Ingeniería Eléctrica. En la Tabla 6 se muestran las palabras que han sido utilizadas con mayor frecuencia en el período 1999-2018, analizadas por quinquenio, como en los casos anteriores. Se puede observar cómo las palabras "sistema", "diseño", "control", "implementación" y "desarrollo" son las palabras más frecuentemente utilizadas. Las nubes de palabras fueron generadas utilizando el sistema disponible libremente en https://www. nubedepalabras.es.

TABLA 6

Frecuencia absoluta de las palabras en los títulos de los documentos presentados como proyecto eléctrico por quinquenios, extraídos del campo "título" en la base de datos del SIBDI, Escuela de Ingeniería Eléctrica 1999-2018.

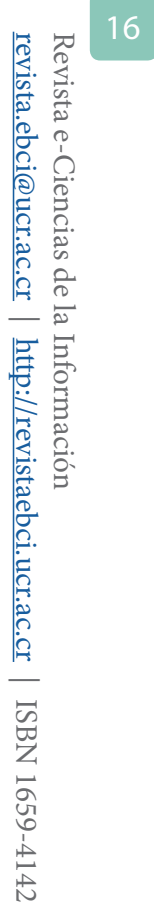

\begin{tabular}{|c|c|c|c|c|c|}
\hline PALABRA & $1999-2003$ & 2004-2008 & 2009-2013 & 2014-2018 & TOTAL \\
\hline sistema & 40 & 56 & 64 & 52 & 212 \\
\hline diseño & 35 & 35 & 51 & 41 & 162 \\
\hline control & 31 & 23 & 50 & 29 & 133 \\
\hline implementación & 20 & 25 & 39 & 37 & 121 \\
\hline desarrollo & 11 & 21 & 28 & 28 & 88 \\
\hline análisis & 13 & 16 & 40 & 18 & 87 \\
\hline estudio & 7 & 13 & 24 & 20 & 64 \\
\hline redes & 7 & 14 & 21 & 9 & 51 \\
\hline distribución & 7 & 8 & 19 & 14 & 48 \\
\hline energía & 8 & 8 & 15 & 13 & 44 \\
\hline laboratorio & 4 & 23 & 12 & 5 & 44 \\
\hline potencia & 3 & 4 & 20 & 17 & 44 \\
\hline interfaz & 11 & 5 & 12 & 12 & 40 \\
\hline tensión & 1 & 5 & 19 & 10 & 35 \\
\hline generación & 2 & 7 & 10 & 15 & 34 \\
\hline controladores & 9 & 9 & 8 & 7 & 33 \\
\hline pid & 11 & 9 & 10 & 1 & 31 \\
\hline pruebas & 6 & 6 & 8 & 10 & 30 \\
\hline datos & 5 & 4 & 11 & 9 & 29 \\
\hline aplicación & 3 & 6 & 11 & 9 & 29 \\
\hline simulador & 1 & 1 & 18 & 9 & 29 \\
\hline aplicación & 3 & 6 & 11 & 9 & 29 \\
\hline señales & 8 & 3 & 11 & 4 & 26 \\
\hline transmisión & 5 & 4 & 9 & 6 & 24 \\
\hline comunicación & 5 & 9 & 7 & 1 & 22 \\
\hline plataforma & 1 & 2 & 8 & 11 & 22 \\
\hline transformadores & 3 & 4 & 9 & 5 & 21 \\
\hline identificación & 11 & 3 & 3 & 1 & 18 \\
\hline
\end{tabular}




\begin{tabular}{|l|c|c|c|c|c|}
\hline \multicolumn{1}{|c|}{ PALABRA } & 1999-2003 & $\mathbf{2 0 0 4 - 2 0 0 8}$ & $\mathbf{2 0 0 9 - 2 0 1 3}$ & $\mathbf{2 0 1 4 - 2 0 1 8}$ & TOTAL \\
\hline software & 3 & 0 & 13 & 2 & 18 \\
\hline medición & 2 & 6 & 9 & 1 & 18 \\
\hline modelado & 2 & 5 & 1 & 10 & 18 \\
\hline prototipo & 1 & 3 & 2 & 10 & 16 \\
\hline calidad & 0 & 11 & 2 & 2 & 15 \\
\hline internet & 3 & 9 & 1 & 1 & 14 \\
\hline robot & 1 & 8 & 0 & 4 & 13 \\
\hline hardware & 2 & 0 & 9 & 1 & 12 \\
\hline
\end{tabular}

Fuente: Elaboración propia, 2019.

Estas palabras son un reflejo del área de acción de la Ingeniería Eléctrica, las cuales aparecen con mucha frecuencia en todos los quinquenios, de forma semejante a "desarrollo" y "análisis". Algunas palabras como "internet" aparecieron con frecuencia durante los dos primeros quinquenios, pero casi desaparecieron los siguientes. Esto se debe a que posiblemente como concepto novedoso fue de interés para desarrollar sistemas o aplicaciones que lo aprovechaban, pero más reciente, con su uso más extendido, no represente una palabra de interés para destacar en el título.

Algunas palabras como "potencia" y "prototipo" han tenido un incremento considerable del uso durante los últimos diez años, en contraposición con otras como "identificación", las cuales se han visto reducidas a lo largo del tiempo.

El cambio que han tenido estas palabras se ve ilustrado en la Figura 3 y Figura 4, en las cuales las nubes de palabras de títulos destacan "control", "sistema" y "CPUCR" en el quinquenio 1999-2003, mientras que en el período 2014-2018 las que más se visibilizan son "sistema", "desarrollo"y "diseño".

\section{FIGURA 3}

Nubes de palabras de los títulos de los proyectos eléctricos de Ingeniería Eléctrica, en el período 1999-2003.

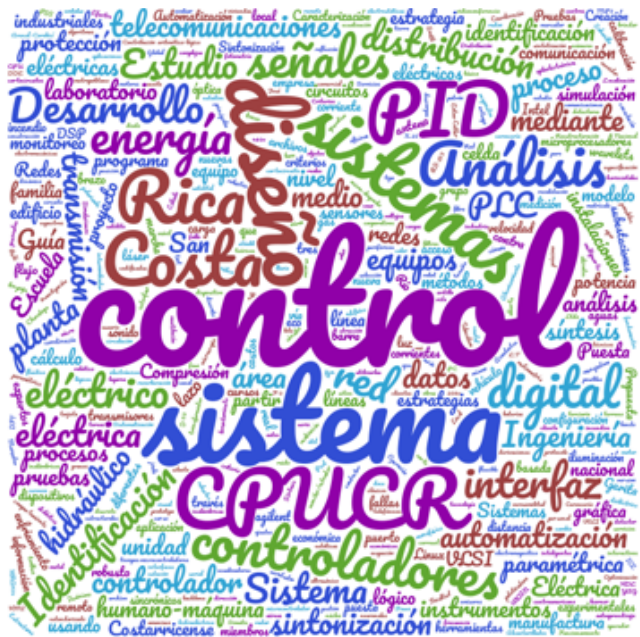

Fuente: Elaboración propia, 2019 
FIGURA 4

Nubes de palabras de los títulos de los proyectos eléctricos de Ingeniería Eléctrica, en el período 2014-2018.

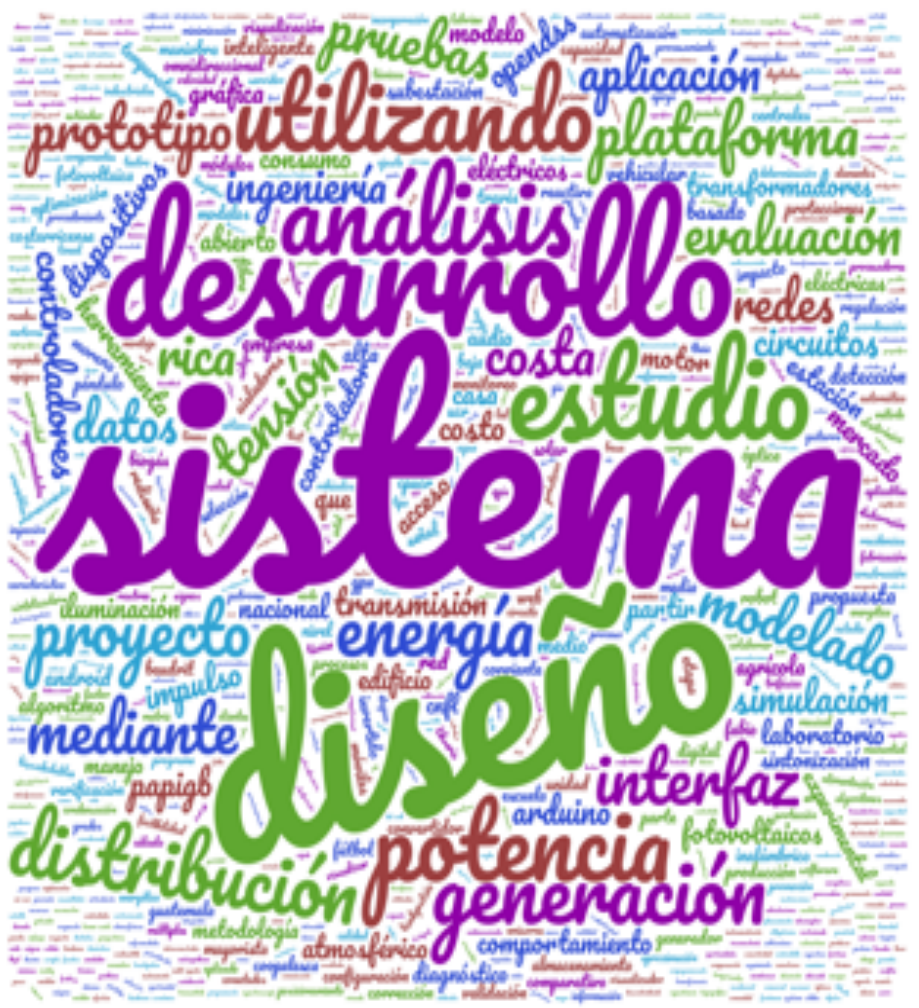

Fuente: Elaboración propia, 2019

Dado que en este estudio se ha realizado un análisis de un tipo de específico de documentos (proyectos finales de graduación) de una carrera específica en un grado académico determinado, como lo es Bachillerato en Ingeniería Eléctrica, la poca disponibilidad de estudios semejantes en la región no permite realizar comparaciones muy directas con los resultados aquí presentados. En especial, cuando no se tiene acceso a todos los datos para establecer comparaciones estadísticas.

Sin embargo, la descripción cuantitativa realizada en el presente trabajo permite realizar algunas comparaciones incipientes sobre aspectos como la longitud promedio de los documentos, que en un primer análisis lo hace equivalente a otros trabajos de graduación en áreas de la ingeniería de América Latina, y una menor participación de las mujeres, también en comparación con otras áreas de la ingeniería en la región.

En cuanto a las materias, existen algunas áreas de la Ingeniería Eléctrica que no han sido representadas en estos proyectos, tales como el electromagnetismo, la ingeniería biomédica y el procesamiento de señales (solamente en uno de los quinquenios se encuentra entre los temas principales). Se ha señalado la dependencia del desarrollo de los proyectos a las especialidades del cuerpo docente, así como la clasificación dada a los documentos en las bases de datos del SIBDI. Con estas consideraciones, es posible indicar los resultados 
mostrados son un insumo para evaluar la necesidad de explorar estos énfasis en futuros proyectos y en la formación de especialistas en la Escuela de Ingeniería Eléctrica.

\section{Conclusiones}

En el presente estudio se ha realizado un análisis bibliométrico de los proyectos de graduación para obtener el grado de bachiller en Ingeniería Eléctrica de la Universidad de Costa Rica. Se analizó un total de 938 documentos, extraídos de la base de datos del sistema OPAC del Sistema de Bibliotecas, Documentación e Información de la Universidad de Costa Rica. Para este fin, desarrollaron algunas rutinas de programación para automatizar parte de los procesos de análisis.

Entre los principales resultados se encuentran una mayor participación de las mujeres en la elaboración de estos documentos, y por ende en el porcentaje de personas graduadas de esta carrera. A lo largo de los años los documentos tienden a ser más concisos en cantidad de páginas.

En cuanto a las temáticas, tanto analizando las materias de clasificación como los títulos, los resultados son un reflejo de las áreas de interés y actividad principal de la Escuela de Ingeniería Eléctrica, así como de la evolución de éstas. Estos resultados pueden constituir un insumo de interés para cuantificar el trabajo e impacto social de la Escuela de Ingeniería Eléctrica en la sociedad costarricense, la actualización de sus temáticas y la evolución de la tecnología.

Por otra parte, el establecimiento de los resultados permitiría contrastarlos con estudios semejantes que puedan llevarse a cabo en otras áreas de la institución, del país en general o de otras instituciones que impartan una carrera de Ingeniería Eléctrica con áreas afines a la Universidad de Costa Rica.

\section{Referencias}

Aguilar, M.C., López, W., Barreto, I., Bolena, Z., Rodríguez, C. y Vargas, E.C. (2007). Análisis bibliométrico de los trabajos de grado del área organizacional de la Facultad de Psicología de la Universidad Santo Tomás. Diversitas: Perspectivas en Psicología, 3(2), 317-334. doi: https://doi.org/10.15332/s1794-9998.2007.0002.11

Ardanuy Baró, J. (2012). La base de datos scopus y otros e-recursos del CBUES como instrumento de gestión de la actividad investigadora. En Breve introducción a la bibliometría. Barcelona, España: Universitat de Barcelona.

Castillo, A. y Xifra, J. (2006). Investigación bibliométrica de las tesis 
doctorales españolas sobre relaciones públicas (1965-2005). Anàlisi: quaderns de comunicació i cultura, 34, 141-161.

Chamba-Herrera, L. (2018). Análisis bibliométrico de las tesis de ingeniero forestal de la Universidad Nacional de Loja, 2013-2017. Bosques Latitud Cero, 8(2), 107-117.

De León-Camero, M. J., Solórzano-Muñoz, C., \& Valencia, G. E. (2018). Análisis bibliométrico de la educación en ingeniería desde el año 2007 a 2017. Revista Educación en Ingeniería, 13(26), 71-76. doi: http://dx.doi. org/10.26507/rei.v13n26.922

Ferrada, C., Díaz-Levicoy, D. A., Salgado Orellana, N., y Puraivan, E. (2019). Análisis bibliométrico sobre educación STEM. Espacios, 40(8), 2-13.

Ferrer-Villalobos, M., Freire-Constante, L., Suasnavas-Bermúdez, P., MerinoSalazar, P., y Gómez-García, A. (2017). Análisis bibliométrico de los artículos originales publicados en la Revista Ciencia \& Trabajo: 19992015. Ciencia \& trabajo, 19(59), 81-85. doi: http://dx.doi.org/10.4067/ $\underline{\text { S0718-24492017000200081 }}$

Garfield, E., y Merton, R. K. (1979). Citation indexing: Its theory and application in science, technology, and humanities. New York: Wiley.

Lagos, R.I. y Pérez-Gutiérrez, M. (2016). Análisis bibliométrico de las tesis de pregrado: el caso de Pedagogía en Educación Física de la Universidad Autónoma de Chile (2007-2012). Revista Ibero-americana de Educação, 70(1), 181-200.

Delgado, E.D., Torres-Salinas, D., Jiménez-Contreras, E. y Ruiz-Pérez, R. (2006). Análisis bibliométrico y de redes sociales aplicado a las tesis bibliométricas defendidas en España (1976-2002): temas, escuelas científicas y redes académicas. Revista española de documentación científica, 29(4), 493-524.

Mathews, B.S. (2004). Internet Resources: Gray literature: Resources for locating unpublished research. College \& Research Libraries News, 65(3), 125-129.

Maz-Machado, A., Jiménez-Fanjul, N.N. y Villarraga-Rico, M.E. (2016). La 
producción científica colombiana en SciELO: un análisis bibliométrico. Revista Interamericana de bibliotecología, 39(2), 111-119.

Moreno-Fernández, O., y Moreno-Crespo, P. (2016). Análisis bibliométrico de las tesis doctorales españolas indexadas con el descriptor "Sector de la educación" (1976/2014). Revista Española de Documentación Científica, 39(3), 1-14. doi: http://dx.doi.org/10.3989/redc.2016.3.1331

Nicaragua, R. N. (2013). Análisis bibliométrico de la producción científica del Centro de Investigaciones Geofísicas (CIGEFI) de la Universidad de Costa Rica en revistas científicas desde 1979 hasta 2011. e-Ciencias de la Información, 3(2), 1-16. doi: 10.15517/ECI.V3I2.10653

Pineda, R.E., Valencia, G. y Roldán, J. (2018). Un análisis bibliométrico de la investigación de sistemas de seguimiento solar. Prospectiva, 16(2), 3540. doi: https://doi.org/10.15665/rp.v16i2.841

Price, D. (1965). Networks of scientific papers. Science, 149, 510-515. doi: 10.1126/science.149.3683.510

Repiso. R., Torres, D. y Delgado, E. (2011). Análisis bibliométrico y de redes sociales en tesis doctorales españolas sobre televisión (1976/2007). Comunicar: Revista científica iberoamericana de comunicación y educación, 19(37), 151-159. doi: http://dx.doi.org/10.3916/C37-2011$\underline{03-07}$

Rey, C.A., y Acevedo, A. (2015). Análisis bibliométrico de las tesis de pregradoy postgrado realizadas en Bogotá, sobre la implementación y evaluación de programas de terapia y modificación del comportamiento. Acta Colombiana de Psicología, 14, 97-111.

Romero Chacón, J.A. (2018). Programa del curso IE-0499 Proyecto Eléctrico, II ciclo 2018. Escuela de Ingeniería Eléctrica, Universidad de Costa Rica, San José, Costa Rica.

Rueda-Clausen, C.F., Villa-Roel, C. y Rueda-Clausen, C.E. (2005). Indicadores bibliométricos: origen, aplicación, contradicción y nuevas propuestas. MedUNAB, 8(1), 29-36.

Salas, G., Ponce, F. P., Méndez-Bustos, P., Vega-Arce, M., Pérez, M. D. L. A., 
López-López, W. y Cárcamo-Vásquez, H. (2017). 25 años de Psykhe: un análisis bibliométrico. Psykhe (Santiago), 26(1), 1-17. doi: http://dx.doi. org/10.7764/psykhe.26.1.1205

Valle, R., y Salvador, E. (2009). Análisis bibliométrico de las tesis de pregrado de la Facultad de Medicina de la Universidad Nacional Mayor de San Marcos. In Anales de la Facultad de Medicina, 70(1), 11-18. 


\section{e-Ciencias de la Información}

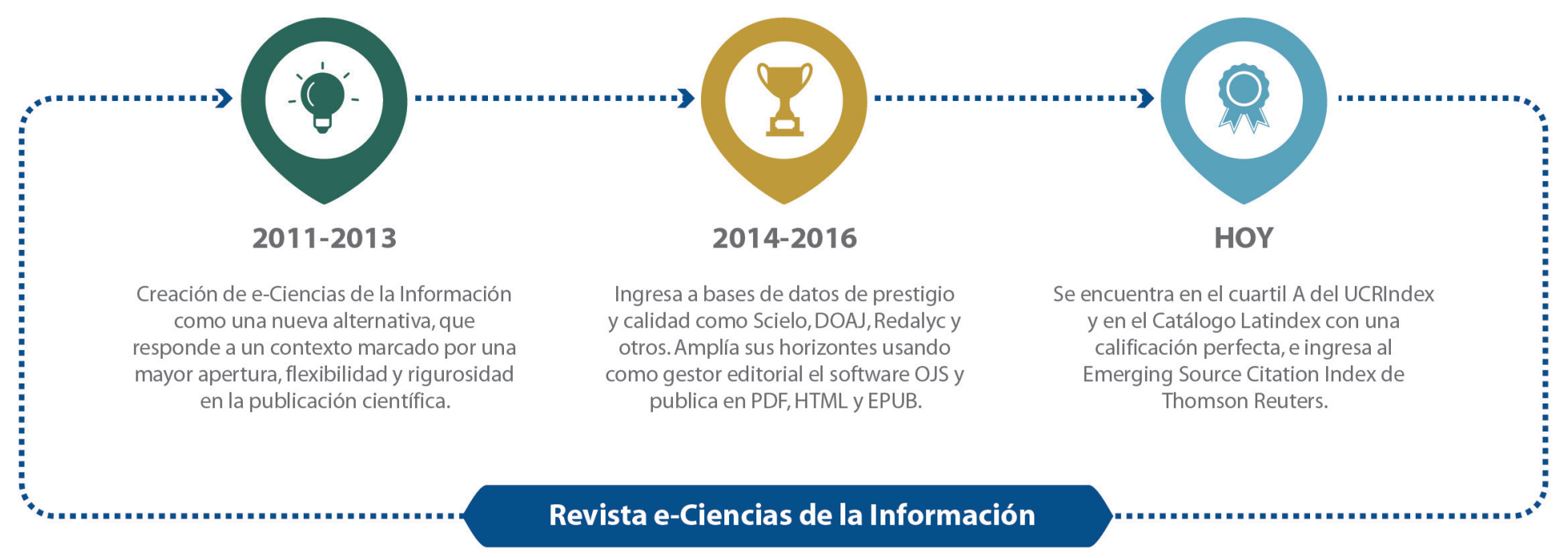

¿Dónde se encuentra idexada e-Ciencias de la Información?

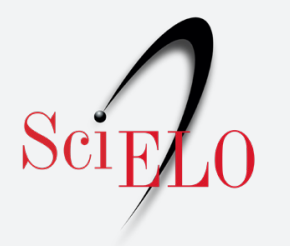

\section{latindex 12 clarivate Analytics}

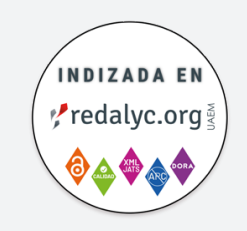

Para más información ingrese a nuestra lista completa de indexadores

¿Desea publicar su trabajo?

Ingrese aquí

O escríbanos a la siguiente dirección revista.ebci@ucr.ac.cr 